\title{
Alterations of Gut Microbiota After Biliopancreatic Diversion with Duodenal Switch in Wistar Rats
}

\author{
Paulette Mukorako ${ }^{1}$ (D) Carlos Lopez ${ }^{1}$. Elena-Dana Baraboi ${ }^{1}$ - Marie-Claude Roy ${ }^{1}$. Julie Plamondon ${ }^{1}$. \\ Natacha Lemoine ${ }^{1} \cdot$ Laurent Biertho $^{1} \cdot$ Thibault V. Varin $^{1} \cdot$ André Marette $^{1} \cdot$ Denis Richard $^{1}$
}

Published online: 5 June 2019

(C) The Author(s) 2019

\begin{abstract}
Background The biliopancreatic diversion with duodenal switch (BPD/DS) represents the most effective surgical procedure for the treatment of severe obesity and associated type 2 diabetes. The mechanisms whereby BPD/DS exerts its positive metabolic effects have however yet to be fully delineated. The objective of this study was to distinguish the effects of the two components of BPD/DS, namely the sleeve gastrectomy (SG) and the DS derivation, on gut microbiota, and to appraise whether changes in microbial composition are linked with surgery-induced metabolic benefits.

Methods BPD/DS, DS, and SG were performed in Wistar rats fed a standard chow diet. Body weight and energy intake were measured daily during 8 weeks post-surgery, at which time glucagon-like peptide 1 (GLP-1), peptide tyrosine tyrosine (PYY), insulin, and glucose were measured. Fecal samples were collected prior to surgery and at 2 and 8 weeks post-surgery. Intraluminal contents of the alimentary, biliopancreatic, and common limbs (resulting from BPD/DS) were taken from the proximal portion of each limb. Fecal and small intestinal limb samples were analyzed by 16S ribosomal RNA gene sequencing.

Results BPD/DS and DS led to lower digestible energy intake ( $P=0.0007$ and $P=0.0002$, respectively), reduced weight gain $(P<0.0001)$ and body fat mass $(P<0.0001)$, improved glucose metabolism, and increased GLP-1 $(P=0.0437$, SHAM versus DS $)$ and PYY levels $(P<0.0001)$. These effects were associated with major alterations of both the fecal and small intestinal microbiota, as revealed by significant decrease in bacterial richness and diversity at $2(P<0.0001$, Chaol index; $P<0.0001$, Shannon index $)$ and 8 weeks ( $P=0.0159$, SHAM versus DS, Chaol index; $P=0.0219$, SHAM versus DS, $P=0.0472$, SHAM versus BPD/DS, Shannon index) post-surgery in BPD/DS and DS, and increased proportions of Bifidobacteriales (a $60 \%$ increase in both groups) but reduced Clostridiales (a 50\% decrease and a 90\% decrease respectively), which were mostly accounted at the genus level by higher relative abundance of Bifidobacterium in both the fecal and intestinal limb samples, as well as reduced abundance of Peptostreptococcaceae and Clostridiaceae in the small intestine. Those effects were not seen in SG rats.

Conclusion The metabolic benefits following BPD/DS are seemingly due to the DS component of the surgery. Furthermore, BPD/DS causes marked alterations in fecal and small intestinal microbiota resulting in reduced bacterial diversity and richness. Our data further suggest that increased abundance of Bifidobacterium and reduced level of two Clostridiales species in the gut microbiota might contribute to the positive metabolic outcomes of BPD/DS.
\end{abstract}

Keywords Bariatric surgery · Body weight · Body fat · Glucose metabolism · Gastrointestinal hormones · Glucagon-like peptide 1 $($ GLP-1) · Peptide tyrosine tyrosine (PYY) · Bifidobacteriales $\cdot$ Clostridiales

Electronic supplementary material The online version of this article (https://doi.org/10.1007/s11695-019-03911-7) contains supplementary material, which is available to authorized users.

Denis Richard

denis.richard@ criucpq.ulaval.ca

1 Institut Universitaire de Cardiologie et de Pneumologie de Québec, Pavillon Marguerite-d'Youville 2725 chemin Sainte- Foy, Québec G1V 4G5, Canada
Bariatric surgery represents the most effective treatment of severe obesity [1]. Among the procedures currently used, vertical sleeve gastrectomy (SG), Roux-en-Y gastric bypass (RYGB), and biliopancreatic diversion with duodenal switch (BPD/DS) have all proved to efficiently reduce excess fat and resolve, to various degrees, obesity-associated co-morbidities such as type 2 diabetes (T2D). RYGB and SG are currently the most performed surgeries [2]. They are preferred over BPD/DS, which has been questionably 
associated with malnutrition [3]. BPD/DS is however the most efficacious surgery to reduce excess fat loss and to alleviate T2D [4]. It is a two-stage procedure including a SG and a biliopancreatic derivation component (duodenal switch (DS)). DS brings the biliopancreatic secretions to the distal ileum and thereby causes malabsorption. Recent data obtained in laboratory rats have demonstrated that the effects of BPD/DS are largely attributable to the DS or malabsorptive component of the surgery $[5,6]$.

The beneficial effects of bariatric surgery have been inevitably associated with gut alterations [7]. The surgery increases the secretion of gut hormones GLP-1 and PYY, which are hormones known to reduce food intake and, as for GLP-1, to produce incretin effects $[8,9]$. In addition, bariatric surgery also appears to induce favorable changes on the gut microbiota [10-12]. For instance, RYGB increases the amount of Gammaproteobacteria whereas it decreases that of Clostridia $[13,14]$ thereby creating a microbiota potentially favoring weight loss and improving metabolic health [14, 15]. Despite recent progress, our current understanding of the impact of bariatric surgery on the gut microbiota is still scarce, especially with regard to certain types of surgery such as BPD/ DS. The present study aimed to address the effects of BPD/DS on the gut microbiota and to distinguish the respective effects of the two components of the BPD/DS, namely SG and DS (referring to the derivation per se). We therefore analyzed the gut microbiota in SHAM, SG, DS, and BPD/DS groups of rats. We hypothesized that (i) the gut microbiota changes over time after BPD/DS, (ii) the two components of BPD/DS have different impacts on gut microbiota (as they have different impacts on energy balance), and (iii) there are distinctive changes in the gut microbiota at different levels of the intestine.

\section{Materials and Methods}

\section{Animals}

Male Wistar rats weighting between 415 and $440 \mathrm{~g}$ (Charles River Canada St-Constant, QC, Canada) were housed individually in plastic cages and kept on a 12:12 h light-dark cycle (lights turned on at 0700 hours) at an ambient temperature of $23{ }^{\circ} \mathrm{C} \pm 1{ }^{\circ} \mathrm{C}$. The rats had access to a standard chow diet (2018 Teklad Global 18\% Protein Rodent Diet, Harlan Laboratories, Montreal, QC, Canada; grams per $100 \mathrm{~g}$ : crude protein 18.6 , fat 6.2 , digestible carbohydrate 44.2 , crude fiber 3.5, neutral detergent fiber 14.7, and ash 5.3). The protocol was approved by the Université Laval Animal Care and Use Committee, and animals were cared according to the Canadian Guide for the Care and Use of Laboratory Animals.

\section{Bariatric Surgery Procedures}

The rats were divided into BPD/DS, SG, DS, and SHAM groups. BPD/DS was performed as previously described and illustrated $[5,16]$. BPD/DS consisted of a vertical SG and a DS. SG consisted of the removal of the two-thirds of the stomach to restrict the amount of food ingested. The DS consisted of a reengineering of the intestine to bring the biliopancreatic secretion at the level of the ileum. The intestine was cut $50 \mathrm{~cm}$ above the ileocecal junction, and the proximal part of the intestine was anastomosed at $20 \mathrm{~cm}$ from the ileocecal junction to create a common limb. The distal part of the intestine was anastomosed to the duodenum to create the alimentary limb. The total length of the rat intestine is approximately $125 \mathrm{~cm}$. The absolute lengths of the limbs that were created were as follows: alimentary $\operatorname{limb}=30 \mathrm{~cm}$, common $\operatorname{limb}=20 \mathrm{~cm}$, biliopancreatic limb $\sim 75 \mathrm{~cm}$. This anastomosis was performed using an end-to-side technique at about $1.5 \mathrm{~cm}$ from the pylorus. The duodenum was ligated with silk between the duodenal anastomosis and the biliopancreatic duct to separate the biliopancreatic limb and ensure the channeling of food only into the alimentary limb. In SG animals, only the gastrectomy was performed and, in DS rats, only the derivation was performed. The SHAM surgery consisted of a midline laparotomy, the handling of intestines and stomach, and the suture of the abdominal wall.

At the time of sacrifice, the duodenal closure was verified in DS and BPD/DS rats and only animals maintaining intact closure (absence of food in the biliopancreatic limb) were included in the statistical analyses. The study began with a total of 29 rats. It ended with 21 rats (success rate of 72\%). Two DS rats were sacrificed due to gastroesophageal refluxes, and 4 DS rats died after the surgery. None of the BPD/DS rats died during the protocol but 2 were excluded from the analyses because food was found in the biliopancreatic limb. Twenty-one rats were used for metabolic studies and gut microbiota analysis: DS $n=4, \mathrm{BPD} / \mathrm{DS} n=5, \operatorname{SHAM} n=6$, SG $n=6$.

\section{Weight and Tissue Weight}

Rats were weighted daily throughout the study. They were killed at the end of the eighth postoperative week with an intraperitoneal (ip) overdose of ketamine $(60 \mathrm{mg} / \mathrm{kg})$ and xylazine $(7.5 \mathrm{mg} / \mathrm{kg})$. The liver, spleen, interscapular brown adipose tissue (BAT), and inguinal and retroperitoneal white adipose tissues (iWAT, rpWAT) were then excised and weighted.

\section{Food and Energy Intake}

Food intake was measured daily. The gross energy intake (GEI) was determined by multiplying daily food intake in 
grams by the gross energy density of the diet, which was determined to be $17.3 \mathrm{~kJ} / \mathrm{g}$ by bomb calorimetry (Parr 6100 calorimeter). During this protocol, we did not collect the feces and therefore did not assess the energy lost in the feces. Thus, the digestible energy intake (DEI), which represents GEI minus the energy loss in the feces, was calculated by multiplying GEI on day 28 by DEI/GEI ratio calculated in a previous study, in which rats were subjected to exactly the same conditions as the rats in the present study and in which fecal energy was determined to directly determine DEI [5]. We used the following ratios: $\mathrm{SHAM}=0.84, \mathrm{SG}=0.84, \mathrm{DS}=0.72$; $\mathrm{BPD} / \mathrm{DS}=0.74$.

\section{Plasma Glucose and Hormones}

After $5 \mathrm{~h}$ of fasting, rats were sacrificed and blood was collected by cardiac puncture, using syringes containing ethylenediaminetetraacetic acid (EDTA) 0.5 M. Postprandial levels of the gut hormones and metabolites were measured as described below. In order to inhibit the activity of proteinases, part of the collected blood was mixed with aprotinin $(500 \mathrm{U} /$ $\mathrm{ml})$ and a DPP IV inhibitor ( $10 \mu \mathrm{l} / \mathrm{ml}$ blood) before centrifugation $\left(3500 \mathrm{rpm}\right.$ for $10 \mathrm{~min}$ at $\left.4{ }^{\circ} \mathrm{C}\right)$. Supernatants were transferred in separate tubes and stored at $-80{ }^{\circ} \mathrm{C}$. An Elisa kit was used for the measurement of plasma PYY, insulin (Milliplex® MAP kit, Billerica, MA, USA), and GLP-1 7-36 dosage (Alpco), and the Wako Autokit Glucose for the measurement of plasma glucose (Wako Diagnostics, Mountain View, CA, USA).

\section{Gut Microbiota Analyses}

\section{Fecal Sampling and DNA Extraction}

Fecal samples were collected in a sterile plastic cup, prior to surgery as well as at 2 and 8 weeks after surgery, and stored at $80{ }^{\circ} \mathrm{C}$ pending further processing. Additionally, gut samples were collected at the time of sacrifice. In the BPD/DS- and DS-operated rats, intraluminal content of the alimentary, biliopancreatic, and common limbs were taken from the proximal portion of each limb (the 10 first centimeters of each limb were cut and the luminal content emptied in a sterile cup). In the SHAM- and SG-operated rats, intraluminal contents were obtained from corresponding levels of the small intestine. Then, samples were stored at $-80^{\circ} \mathrm{C}$ until DNA extraction. Genomic DNA was extracted using the FastDNA® Spin Kit for Soil (MP Biomedicals, Solon, OH, USA) following the manufacturer's instruction by using a combination of physical cell-disruption (bead beater) and silica column purification, some modifications were added for better yields. Briefly, approximately $100 \mathrm{mg}$ of feces is manually crushed in sodium phosphate and MT buffers (MP Bio) and mixed with lysing matrix particles. Homogenization is carried out in a MiniBeadBeater
(Bertin instruments, Montigny-le-Bretonneux, France) to break bacterial cells. After centrifugation (10 min, 14,000g), supernatant is treated with a protein precipitation solution (MP Bio) in order to remove proteins present in large quantities in feces. Then, a SEWS-M wash solution (MP Bio) is added to remove impurities once DNA is bound to the binding matrix. After two centrifugations ( $2 \mathrm{~min}, 14,000 \mathrm{~g}$ ), DNA is eluted with $50 \mu \mathrm{l}$ DNase-free water. Total extracted DNA is quantified with a BioDrop spectrophotometer (Montreal Biotech, Kirkland, QC, Canada). All extracted DNA samples were pooled according to treatment conditions (SHAM, SG, DS, BPD/DS), time of sampling (week 0 , week 2,8 , sacrifice), and location (alimentary limb, biliopancreatic limb, common limb, and feces) which resulted in a total of 125 DNA samples.

\section{Statistical Analyses}

Data are presented as means \pm standard errors of the mean (SEM). Statistical comparisons between groups were performed using one-way analysis of variance (ANOVA) followed by Bonferroni with three planned comparisons (SHAM vs $\mathrm{SG}, \mathrm{DS}, \mathrm{BPD} / \mathrm{DS})$. When needed, data were square root transformed to satisfy the variance normality criterion. Body weight and gross energy intake curves were analyzed using two-way ANOVA to determine the main interaction effect of surgery and time (GraphPad Prism version 7.00). Results were considered significant at $P<0.05$.

\section{Results}

\section{Body Weight and Tissues Weights}

From the second to the eighth week post-surgery, BPD/DS and DS rats exhibited significantly lower body weights than SHAM rats $(P<0.0001 ;$ Fig. 1a). The total weight gain for the whole 8 weeks of treatment was also lower for BPD/DS and DS rats compared with SHAM rats $(P<0.0001$; Fig. $1 b)$. SHAM and SG animals did not show any difference in their body weight gains during this period ( $P=0.6517$; Fig. $1 b)$. $\mathrm{BPD} / \mathrm{DS}$ and DS rats also showed significant reductions in their WAT and BAT masses compared with SHAM rats $(P<0.0001$ and $P<0.0001$, respectively; Table 1). Liver and spleen weights in BPD/DS and DS were also reduced compared with SHAM $(P<0.0001$ and $P=0.0002$, respectively; Table 1). There was no difference between SHAM and SG groups in the weights of WAT, BAT, liver, and spleen.

\section{Energy Intake}

During the first week after surgeries, there was no difference in GEI between groups. On days 8 and 14, BPD/DS exhibited a lower GEI than SHAM $(P=0.0027$ and $P<0.0001$; 
a
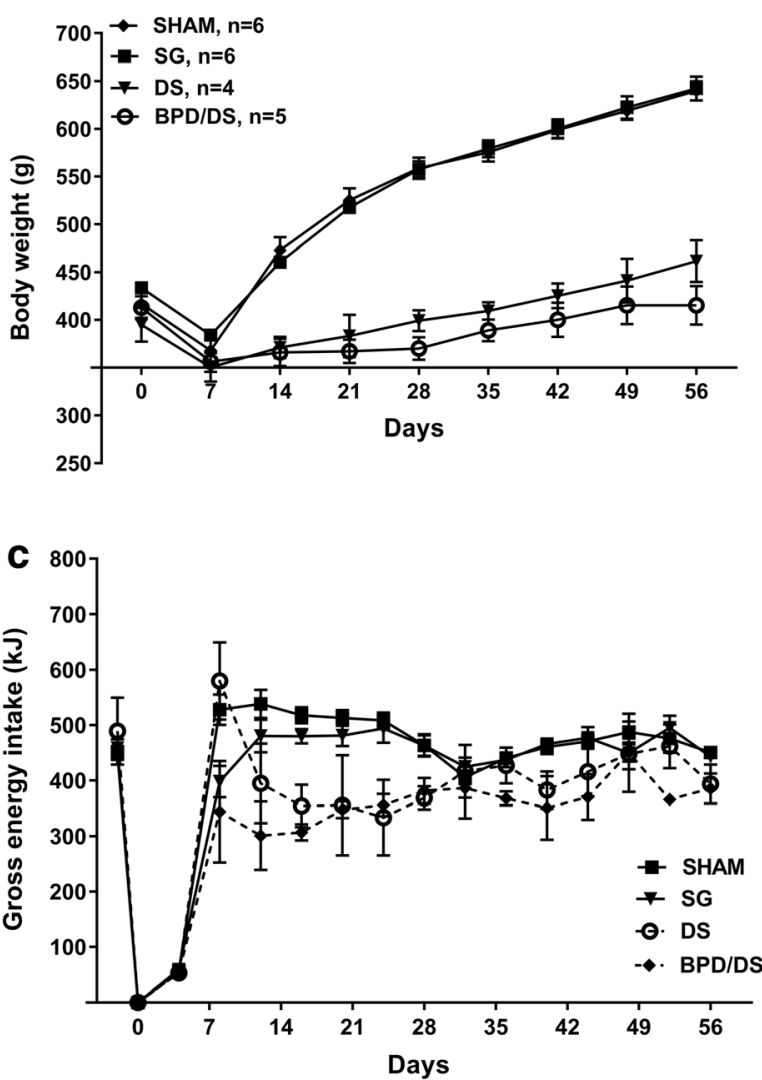

Fig. 1 Effects of BPD/DS, DS, SG, and SHAM surgery on body weight and food intake. Body weight (a) and body weight gain (b) from the day of surgery to the end of 8 weeks post-surgery are shown in the 4 groups fed a chow diet. Gross energy intake (c) prior to the day of surgery to the end of 8 weeks post-surgery is shown and digestible energy intake (d) on day 28 post-surgery. Data are shown as means \pm SEM. $* * * P<0.001$ vs SHAM, $* * * * P<0.0001$ vs SHAM. The DEI on day 28 was calculated by

Fig. 1c). On day 24, DS and BPD/DS showed a significant reduction in GEI compared with $\operatorname{SHAM}(P=0.0083$ and $P=$ 0.0154 , respectively; Fig. 1c). Six weeks after surgery, only BPD/DS showed a significant reduction in GEI compared with SHAM $(P=0.0166$; Fig. $1 c)$. The GEI of SG rats was not different from that of SHAM rats $(P>0.9999)$ except on

Table 1 Effects of BPD/DS, DS, SG, and SHAM surgery on adipose tissues, liver, and spleen on the eighth week post-surgery. Data are shown as means \pm SEM. $* * * * P<0.0001$ vs SHAM

\begin{tabular}{lllll}
\hline & SHAM & SG & DS & BPD/DS \\
\hline iWAT & $7.3 \pm 0.6$ & $7.3 \pm 0.6$ & $2.2 \pm 0.1^{* * * *}$ & $2.0 \pm 0.2^{* * * *}$ \\
rpWAT & $7.3 \pm 0.7$ & $6.9 \pm 1.1$ & $0.6 \pm 0.0^{* * * *}$ & $1.2 \pm 0.2^{* * * *}$ \\
Sum of WATs & $14.6 \pm 1.2$ & $14.2 \pm 1.6$ & $2.8 \pm 0.1^{* * * *}$ & $3.3 \pm 0.4^{* * * *}$ \\
BAT & $0.8 \pm 0.0$ & $0.7 \pm 0.0$ & $0.2 \pm 0.0^{* * * *}$ & $0.2 \pm 0.0^{* * * * *}$ \\
Liver & $22.6 \pm 0.4$ & $22.3 \pm 1.1$ & $15.3 \pm 1.2^{* * * *}$ & $14.9 \pm 0.2^{* * * *}$ \\
Spleen & $1.3 \pm 0.0$ & $1.3 \pm 0.0$ & $0.8 \pm 0.0^{* * * *}$ & $0.8 \pm 0.0^{* * * *}$ \\
\hline
\end{tabular}

$B A T$, brown adipose tissue; $i W A T$, inguinal white adipose tissue; $r p W A T$, retroperitoneal white adipose tissue
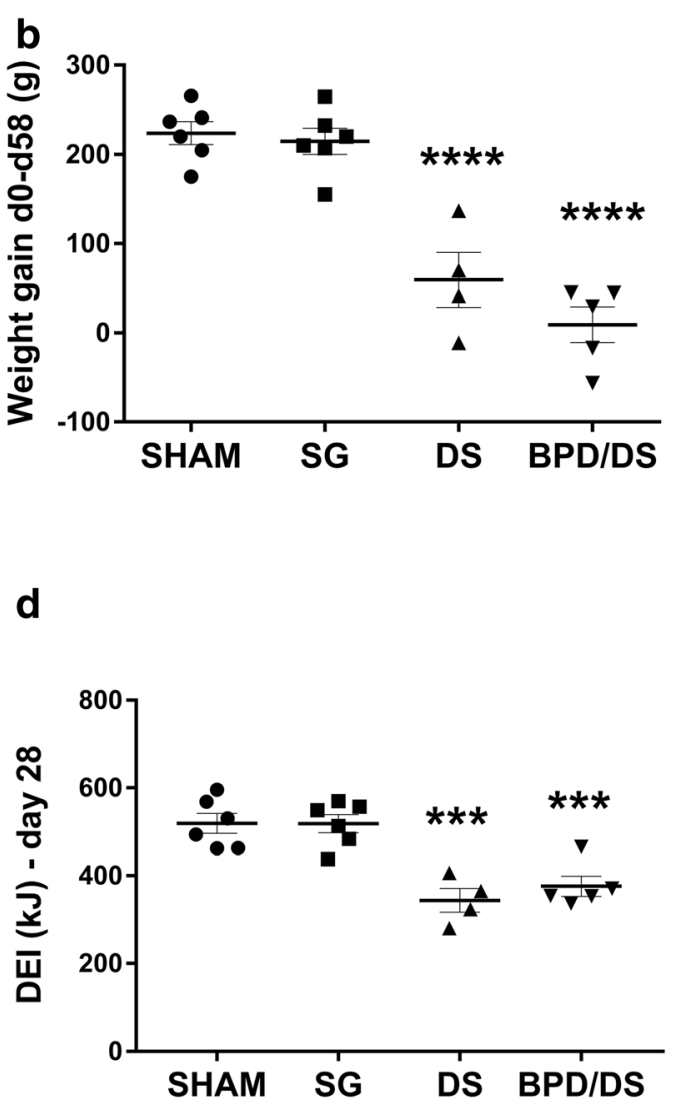

multiplying the GEI on day 28 by DEI/GEI values from a preceding study done in our lab on rats fed a chow diet (Li, Baraboi et al., Surg Obes Relat Dis., 2014): DEI = GEI $\times(\mathrm{DEI} / \mathrm{GEI}), \mathrm{DEI} / \mathrm{GEI} \mathrm{SHAM}=0.84, \mathrm{DEI} / \mathrm{GEI}$ $\mathrm{SG}=0.84, \mathrm{DEI} / \mathrm{GEI} \mathrm{DS}=0.72 \mathrm{DEI} / \mathrm{GEI} \mathrm{BPD} / \mathrm{DS}=0.74$. GEI gross energy intake, DEI digestible energy intake, SG sleeve gastrectomy, DS duodenal switch, BPD/DS biliopancreatic diversion with duodenal switch

day $8(P=0.0001)$. There was no difference in the DEI of SG versus that of SHAM rats $(P>0.9999)$. DEI was lower in DS and BPD/DS than that in SHAM $(P=0.0002$ and $P=0.0007$, respectively; Fig. 1d). This was mostly attributable to the increased fecal energy loss caused by malabsorption in both DS and $\mathrm{BPD} / \mathrm{DS}$ rats [5].

\section{Gastrointestinal Hormones and Glucose Metabolism}

DS rats exhibited increased levels of GLP-1 compared with SHAM rats $(P=0.0437$; Fig. 2a). Additionally, DS and BPD/ DS groups showed higher levels of PYY than SHAM rats $(P<0.0001$ and $P<0.0001$; Fig. 2b). BPD/DS rats had lower levels of insulin than SHAM rats $(P=0.0387$; Fig. $2 c)$ but there was no difference in glucose levels between groups $(P=0.5106$ SHAM versus DS, and $P=0.0538$ SHAM versus BPD/DS; Fig. 2d). HOMA-IR index, calculated for each group, suggested that BPD/DS rats were more sensitive to insulin compared with SHAM rats $(P=0.0051$; Supplementary Fig. 1). 

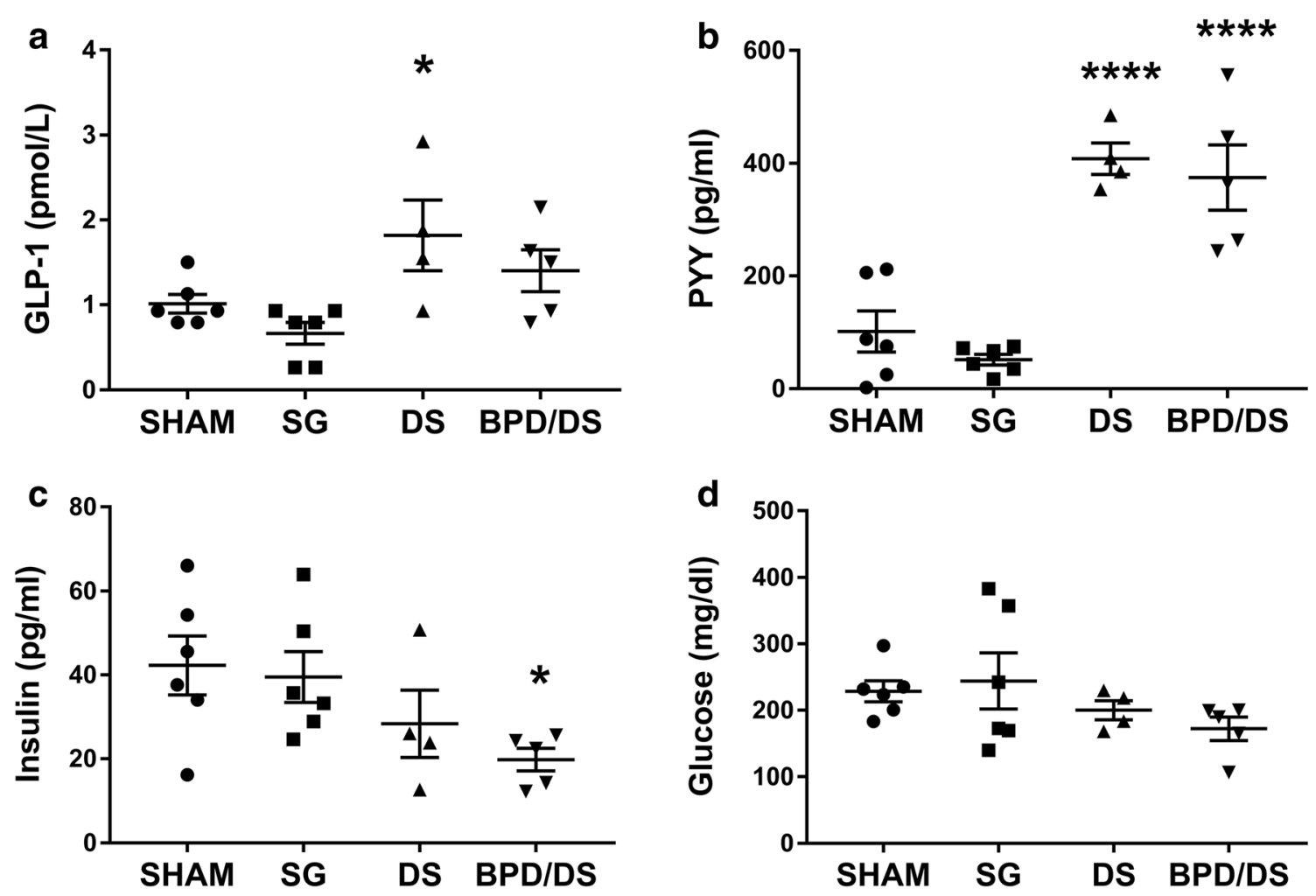

Fig. 2 Effects of BPD/DS, DS, SG, and SHAM surgery on plasma levels of GLP-1 7-36 (a), PYY (b), insulin (c), and glucose (d) on the eighth week post-surgery. $* P<0.05$ vs SHAM, $* * * * P<0.0001$ vs SHAM. Data are means \pm SEM. Comparisons between groups were performed

\section{Composition of Gut Microbiota over Time After DS and BPD/DS Surgeries}

We performed 16S ribosomal RNA gene sequencing on fecal samples collected prior to and at 2 and 8 weeks post-surgery in SHAM, SG, DS, and BPD/DS groups. Analyses of the Chao1 index revealed that the richness of the microbiota was markedly reduced at week 2 post-surgery in DS and BPD/DS $(P<0.0001$ and $P<0.0001$; Fig. 3a) as well as at week 8 in DS group $(P=$ 0.0159 ; Fig. 3a) in comparison with the SHAM control. The Shannon index for both groups also declined at week 2 $(P<0.0001$ and $P<0.0001$; Fig. 3b), indicating lower gut microbiota diversity, and remained lower than SHAM group at week 8 ( $P=0.0219$ and $P=0.0472$; Fig. 3b). Clostridiales was the most abundant order of the fecal microbiota prior to surgery in all groups. At week 2, the relative abundance of Clostridiales was unchanged in SHAM and SG, whereas it was reduced in $\mathrm{DS}$ and $\mathrm{BPD} / \mathrm{DS}$ rats (a $50 \%$ decrease in BPD/DS group and a 90\% decrease in DS rats, compared with SHAM) (Fig. 3c). At week 8, Clostridiales remained in lower proportions in both DS and BPD/DS rat fecal samples (Fig. 3c). Bacteroidales was the second most abundant order in the fecal microbiota of all groups before the surgery ( 25 to $30 \%$ ). At weeks 2 and 8, there was a small decrease in DS and BPD/DS rats (approximately 10 to $15 \%$ decrease in both groups) (Fig. 3c). Meanwhile,

using one-way ANOVA test; GLP-1 and insulin data were square root transformed. SG sleeve gastrectomy, DS duodenal switch, BPD/DS biliopancreatic diversion with duodenal switch, GLP-1 glucagon-like peptide 1, PYY peptide tyrosine tyrosine

Bifidobacteriales, the third most abundant order pre-surgery, was significantly increased at week 2 and week 8 in DS and BPD/DS groups (approximately a $60 \%$ increase in both groups) (Fig. 3c). The increase in Bifidobacteriales noticed at week 2 and week 8 in the DS and BPD/DS groups was mostly due to high proportions of reads assigned to species from the genus Bifidobacterium, while the decrease in Clostridiales was due to a drop in reads assigned to the Peptostreptococcaceae and the Clostridiaceae (Fig. 3d).

\section{Composition of Gut Microbiota by DS and BPD/DS Along the Small Intestine}

We proceeded to the same microbiota analyses for samples collected at the time of sacrifice at different levels of the intestine (alimentary, biliopancreatic, and common limbs). The results indicate that DS and BPD/DS altered the richness specifically in the common limb $(P=0.0074$ and $P=0.0344$; Fig. 4a); the diversity of the bacterial populations in this limb was however not altered as compared with SHAM (Fig. 4b). Clostridiales was the most represented order in alimentary and common limbs from SHAM and SG animals (75 to $90 \%$ ). In contrast, alimentary and common limbs of DS and BPD/DS groups showed a decline in Clostridiales abundance and increased proportions of Bifidobacteriales ( 80 to $85 \%$ ) (Fig. 4c). 
a

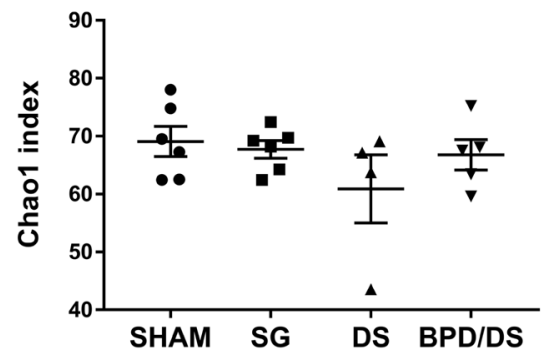

b

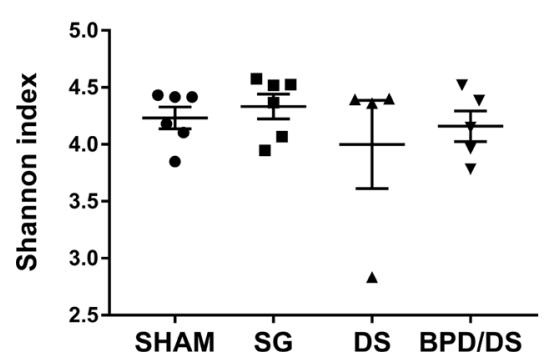

C

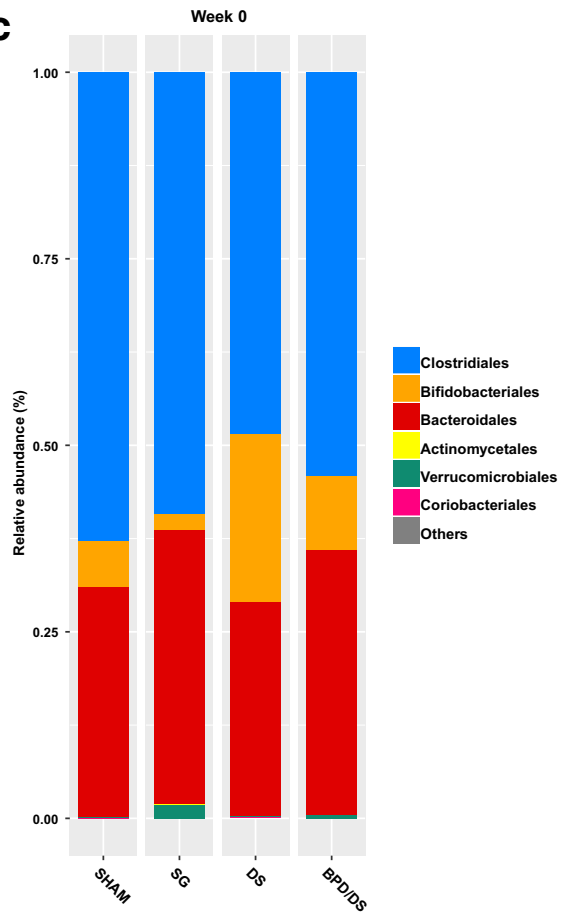

Week 2

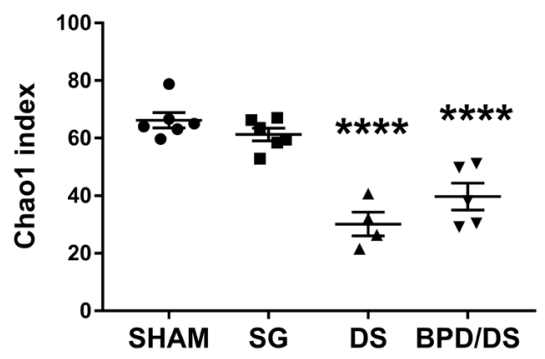

Week 2

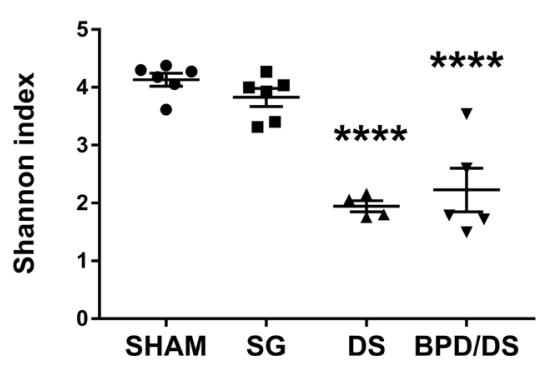

Week 2

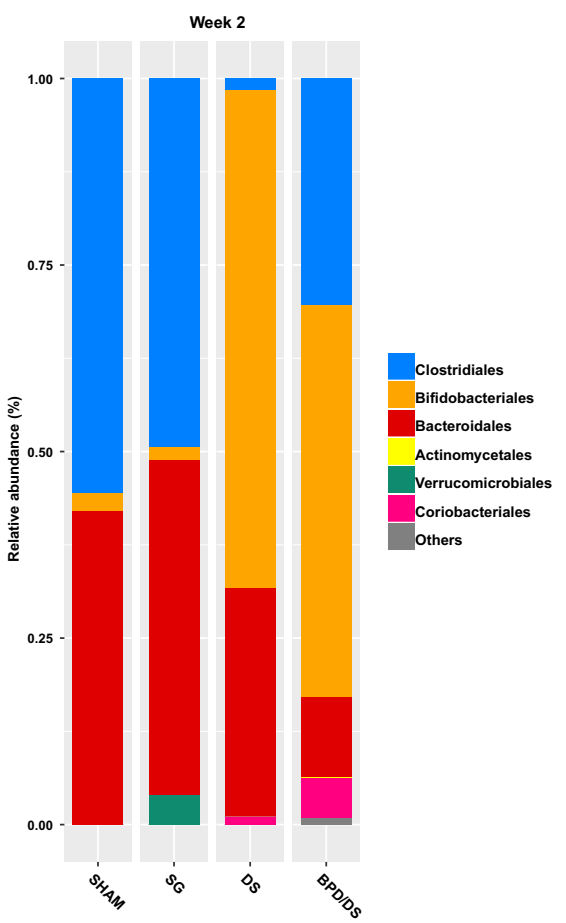

Week 8

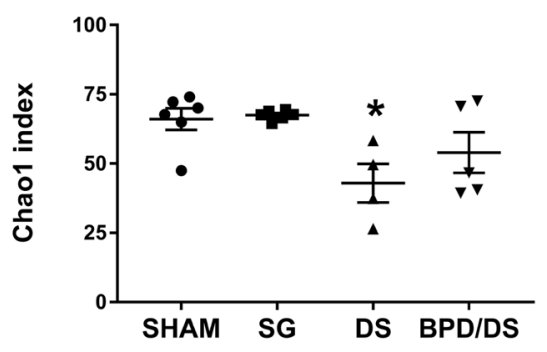

Week 8

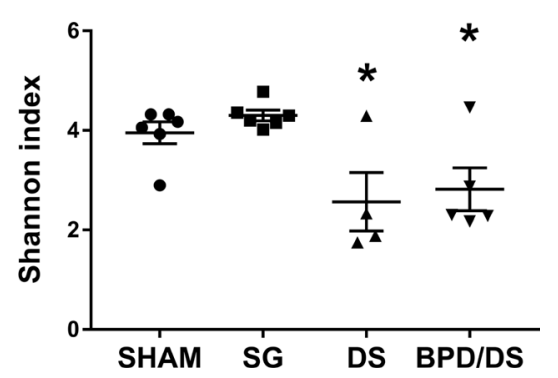

Fig. 3 Richness and diversity of fecal microbiota in SHAM, SG, DS, and BPD/DS groups. Chao1 and Shannon indexes estimate the intrinsic richness and diversity respectively calculated for each group: (a), (b) in feces at different time points. Data are means \pm SEM. $* P<0.05$ vs SHAM, $* * * * P<0.0001$ vs SHAM. c Relative abundance distribution of operational taxonomic unit (OTU) sequences (at the order level) in feces samples prior the surgery (week 0), week 2, and on week 8 in SHAM, SG, DS, and BPD/DS rats. d Statistical comparisons of fecal microbial profiles at the genus level in different types of surgery. Significant differences in the abundance of bacterial genera between surgeries: at week 2 and at week 8 post-surgery. The graphs on the left side exhibit the mean proportion of sequences assigned to each genus. The dot plots on the right side exhibit the difference in mean proportions between surgeries with associated q-values. Error bars on both sides of dots represent the $95 \%$ CIs. Only genera with a q-value $<0.05$ and a difference in mean proportions value $>1$ were considered 
d

$\square$ SHAM_week $2 \square$ BPD/DS_week 2 95\% confidence intervals

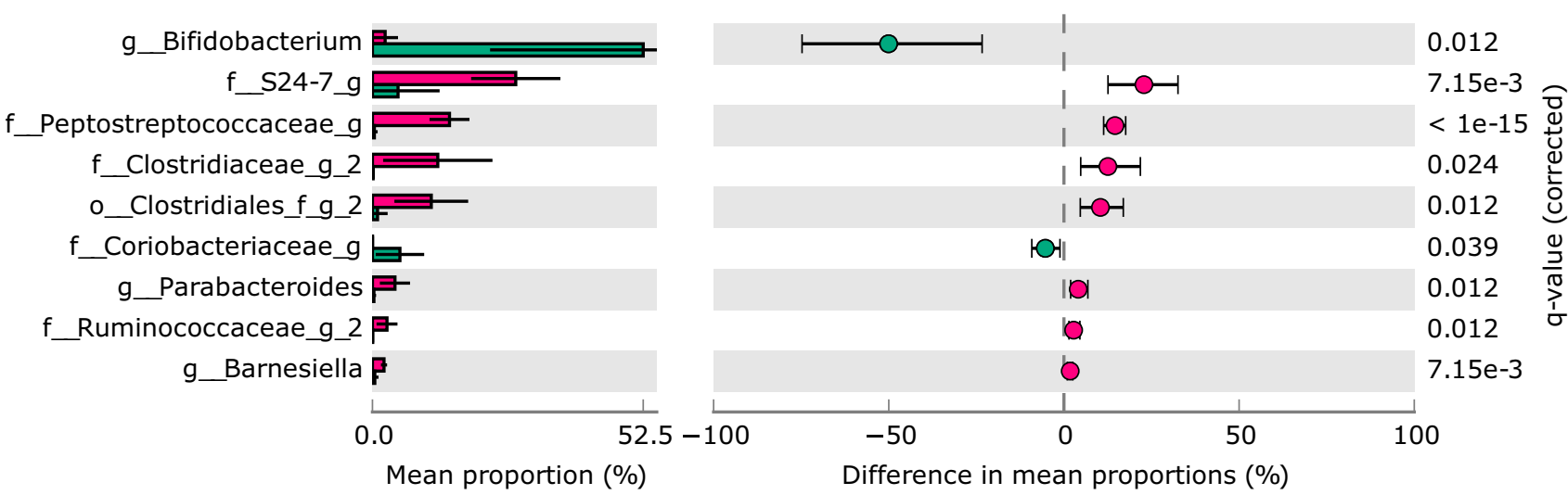

SHAM_week $2 \square$ DS_week 2

$95 \%$ confidence intervals

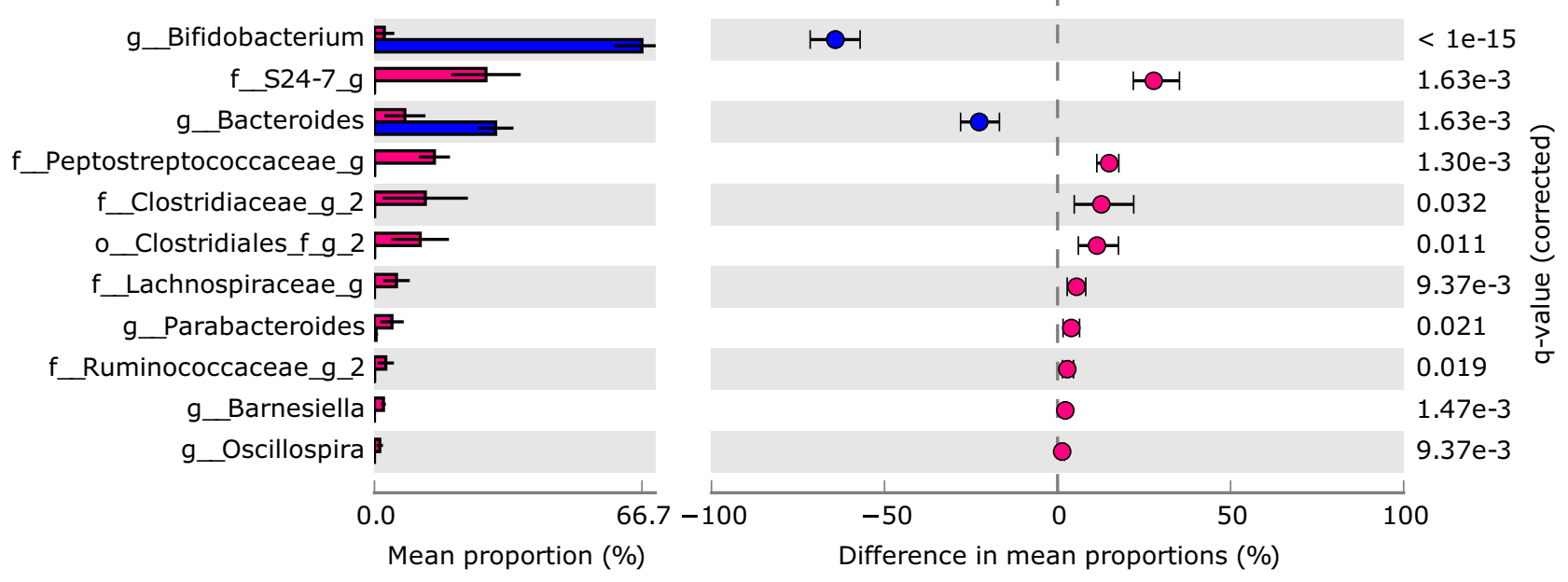

\section{SHAM_week $8 \square$ BPD/DS_week 8 95\% confidence intervals}

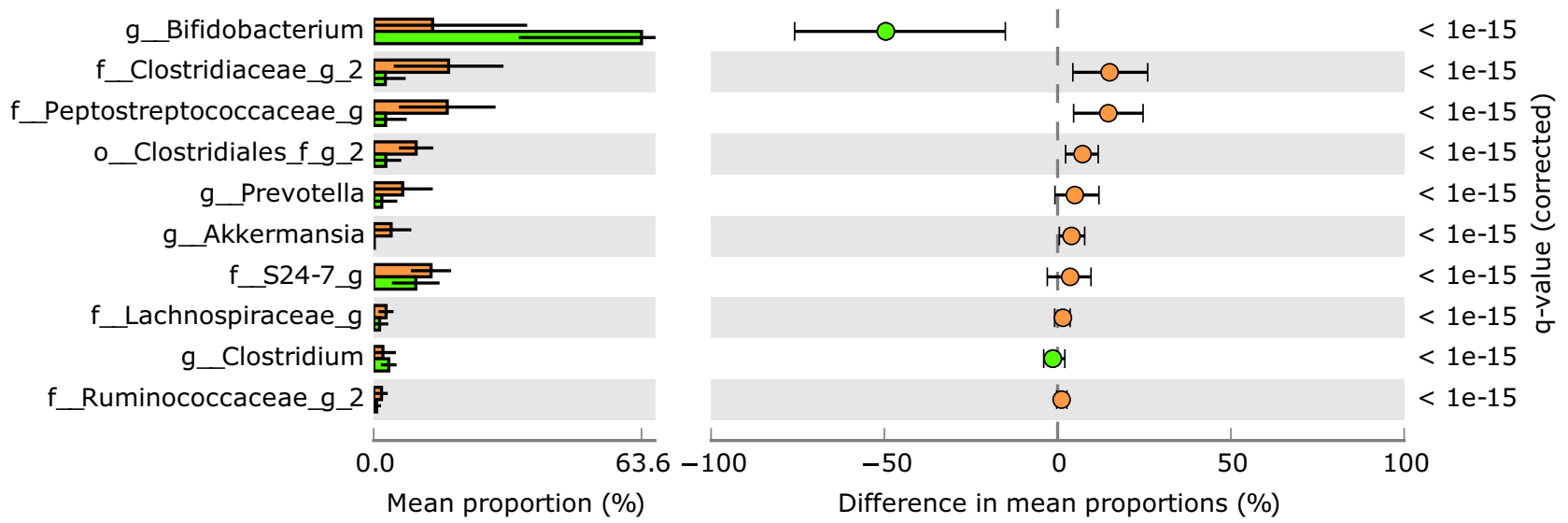

Fig. 3 (continued)

In biliopancreatic limb, Actinomycetales was the most abundant order in SHAM and SG (55\% and 60\% respectively). In the DS group, Actinomycetales, Clostridiales, and Verrucomicrobiales were mostly represented. In addition to these, there was a higher proportion of Bifidobacteriales in this limb following BPD/DS (Fig. 4c). 
At the genus level, DS and BPD/DS promoted significant levels of Bifidobacterium and lower levels of the Peptostreptococcaceae and Clostridiaceae in the common and alimentary limbs as seen in fecal samples (Fig. 4d).

\section{Discussion}

One primary goal of the present study was to examine the effects of the BPD/DS on the gut microbiota in rats, in which BPD/DS largely replicates the beneficial metabolic effects seen in humans $[3,4,17]$. We found that the fecal microbiota of BPD/DS rats significantly differs from that of SHAM animals. A decline in the diversity and richness of gut microbiota was seen in BPD/DS rats, as revealed by the Shannon and the Chaol indices. The effects seemed less apparent at week 8, but nonetheless remained significant except for Chaol in BPD-DS, possibly because of the apparent variability of the scores in the latter group. Furthermore, analysis of the microbial profile demonstrated a major shift from a microbiota dominated by Clostridiales prior to the surgery (or in SHAM rats) to a microbiota highly concentrated in Bifidobacteriales soon post-surgery. Since BPD/DS includes two surgical components, namely the SG and the DS, which distinctly influence energy homeostasis and metabolism $[4,6]$, we also aimed at dissecting the respective contribution of those components on the effect on gut microbiota. We found that DS, similar to BPD/DS, caused marked alterations in the fecal microbiota compared with SHAM or SG surgery. Finally, as the BPD/ DS divides the gut into three functional segments, namely the alimentary, biliopancreatic, and common limbs, we were interested in looking at the microbiota in each of those segments. We observed that the gut microbiota within the alimentary and common limbs mirrored that of the feces as it exhibited a high content in Bifidobacteriales. On the other hand, the microbiota of the biliopancreatic limb differs from that of the two other limbs as it contains a substantial amount of Actinomycetales.

The present results confirmed the ability of certain bariatric surgical procedures to markedly affect the gut microbiota in humans $[18,19]$ as well as in laboratory rodents [20]. Similar to BPD/DS in this study, RYGB in a previous report appeared to lower the diversity of gut microbiota while reducing the proportion of Clostridiales compared with SHAM surgery [21]. However, unlike RYGB, BPD/DS considerably elevated the proportion of Bifidobacteriales as represented by increased abundance of the Bifidobacterium genus. That bariatric procedures may lower the diversity of the gut microbiota while reducing the proportion of Clostridiales has also been reported in humans before $[13,19,22]$.

The mechanisms whereby BPD/DS affects the gut microbiota remain to be fully described. We report here the importance of the malabsorptive component of the BPD/DS in altering the composition of the microbiota. Indeed, the gut microbiota of the DS and BPD/DS rats were comparable and different from that of SG and SHAM rats. SG rats presented a similar microbial composition compared with SHAM rats, in agreement with previous observation [21]. Malabsorption is inherent to the DS component of the BPD/DS surgery and likely explains the presence of a gut microbiota that appears specific to the BPD/DS and different from RYGB [21], which is not described as a priori being a malabsorptive surgery [23]. Our rat BPD/DS procedure has been reported to enhance fecal energy loss and thus cause malabsorption $[5,6]$. That the presence of undigested digestible nutrients in the lower gut can alter the microbiota appears plausible. However, how such a phenomenon could elevate the proportion of the bacteria from the Bifidobacteriales order warrants further investigation. It seems unlikely that the change in the microbiota is attributable to the biliopancreatic secretion per se. In fact, the changes seen in the fecal microbiota were not only seen in the common limb, where the biliopancreatic secretion is derived, but also in the alimentary limb, which is derived from the biliopancreatic secretion. Furthermore, the biliopancreatic limb exhibits a microbiota that differs from that of the alimentary and common limbs.

It was previously reported that bariatric procedures can lead to a pathological bacterial overgrowth in the small intestine referred to as the small intestine bacterial overgrowth (SIBO) syndrome [24]. Such a syndrome was seen most of the times following jejunoileal bypass (JIB) [25], a bariatric procedure that is no longer used, in part because of its inducing effects on bacterial overgrowth. In fact, JIB created a long partially-disconnected segment of the small bowel [26] that emerged as being very propitious for bacterial overgrowth [25]. SIBO has also been reported following RYGB but in a minority of cases [27] and it would seem unlikely that the shifts of bacterial orders seen in this study be due to a pathological bacterial overgrowth. In fact, one can argue that the increase in the Bifidobacteriales in BPD-DS and DS rats in this study was attributable to rather a change in the proportion of bacteria than to bacterial overgrowth.

The changes in the microbiota seen in BPD/DS and DS rats were associated with beneficial effects of the two malabsorptive procedures. Indeed, both BPD/DS and DS in this study reduced fat deposition and improved insulin sensitivity, as previously reported [6] and as suggested from the HOMA-IR values in Supplementary Fig. 1. The SG surgery, as performed in this study, did not induce any change in weight gain, metabolic variables, and gut microbiota. As previously discussed $[5,6]$, the occurrence of metabolic longlasting metabolic effects following SG likely appears to depend on the proportion of the stomach removed. In this study as well as in previous ones [5, 6], we removed $60 \%$ of the stomach as compared with $80 \%$ in studies showing reducing effects of SG on body fat $[28,29]$. Given that changes in the 
a

Alimentary Limb

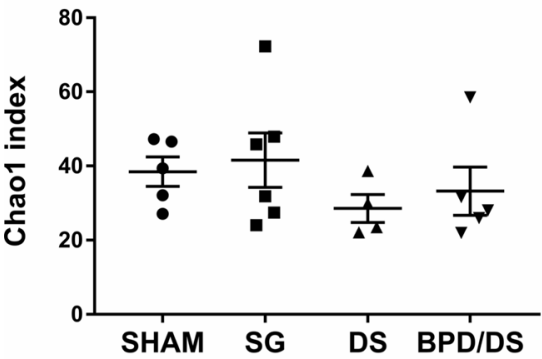

b

Alimentary Limb

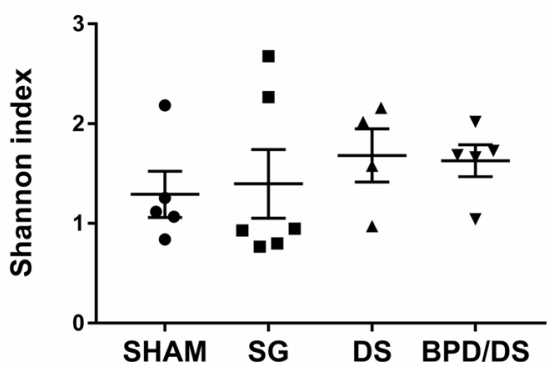

C

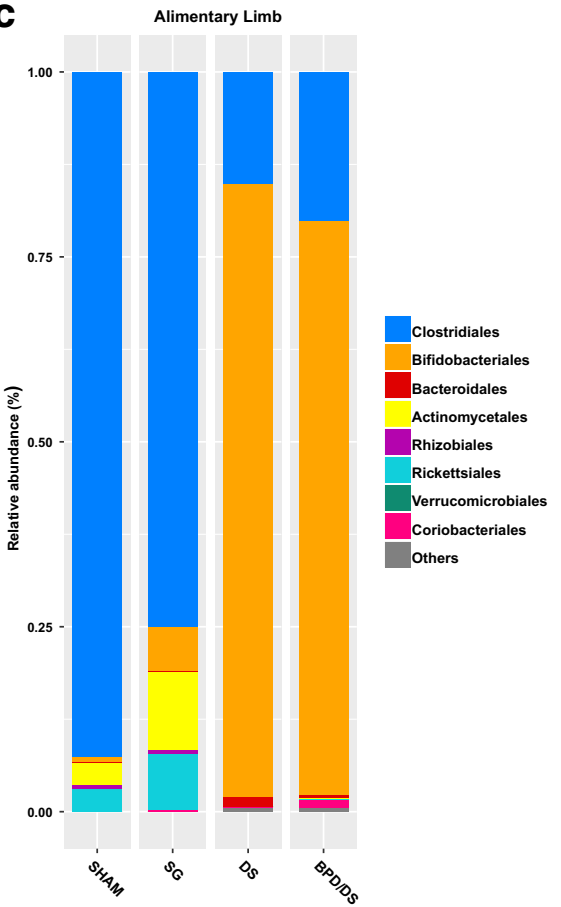

Biliopancreatic Limb

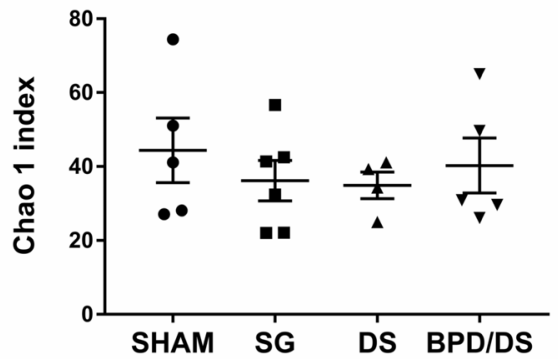

Biliopancreatic Limb
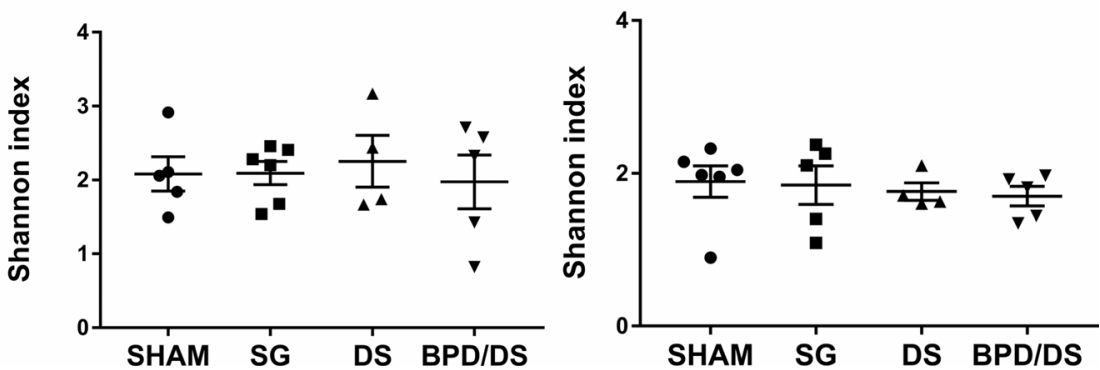

Common Limb
Fig. 4 Richness and diversity of small intestine microbiota in SHAM, SG, DS, and BPD/DS groups. Chaol and Shannon indexes estimate the intrinsic richness and diversity respectively calculated for each group: (a), (b) at the time of sacrifice in each limb. Data are means \pm SEM. $* P<0.05$ vs SHAM, $* * P<0.01$ vs SHAM. c Relative abundance distribution of operational taxonomic unit (OTU) sequences (at the order level) among alimentary, biliopancreatic, and common limbs of SHAM, SG, DS, and BPD/DS rats. d Statistical comparisons of fecal microbial profiles at the
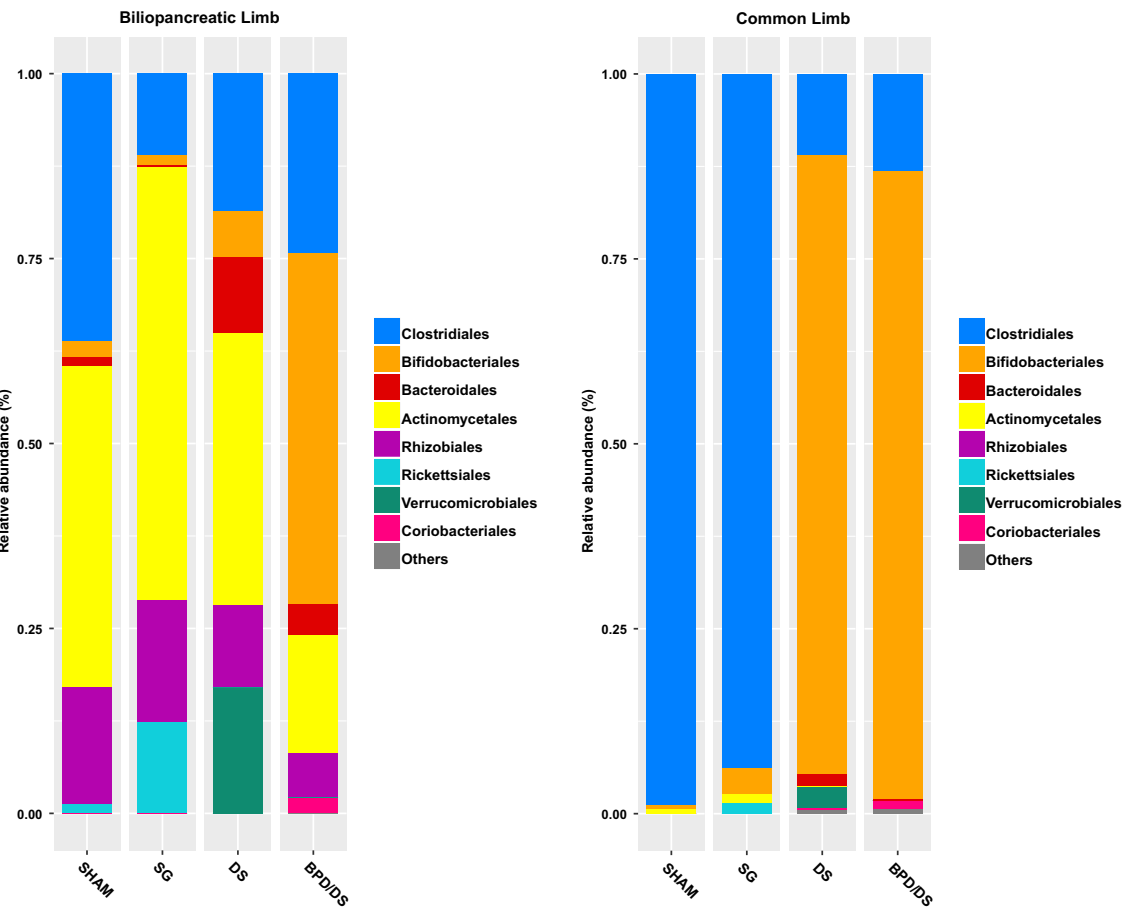

genus level of intestinal segments in different types of surgery. Significant differences in the abundance of bacterial genera between surgeries: in common limb and in the alimentary limb. The graphs on the left side exhibit the mean proportion of sequences assigned to each genus. The dot plots on the right side exhibit the difference in mean proportions between surgeries with associated q-values. Error bars on both sides of dots represent the $95 \%$ CIs. Only genera with a q-value $<0.05$ and a difference in mean proportions value $>1$ were considered 


\section{d}

\section{Common Limb}

SHAM_CL $\square$ BPD/ DS_CL

$\%$ confidence intervals

g_Bifidobacterium

f__Peptostreptococcaceae_g

f_Clostridiaceae_g_2
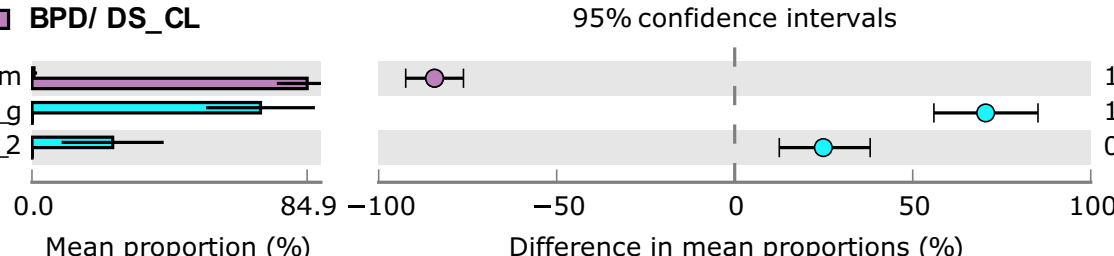

$41 e-3$ $1.41 e-3$ 어 0.017

SHAM_CL $\square$ DS_CL
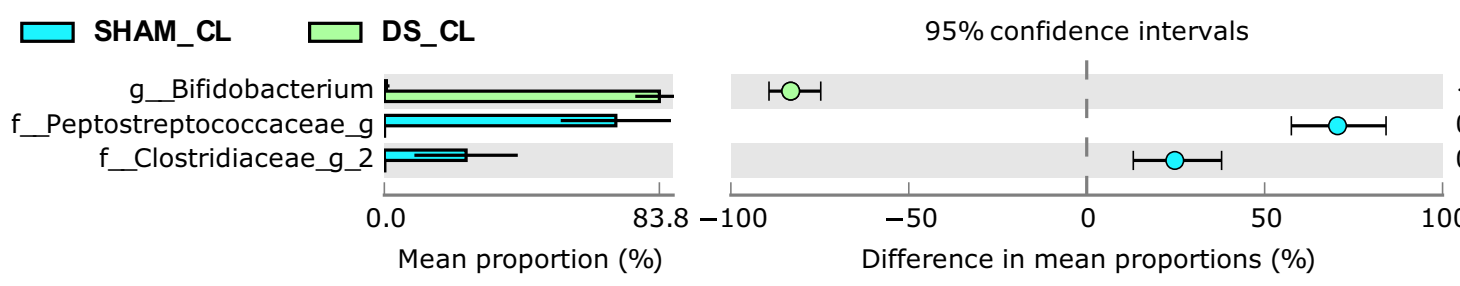

\section{Alimentary Limb}
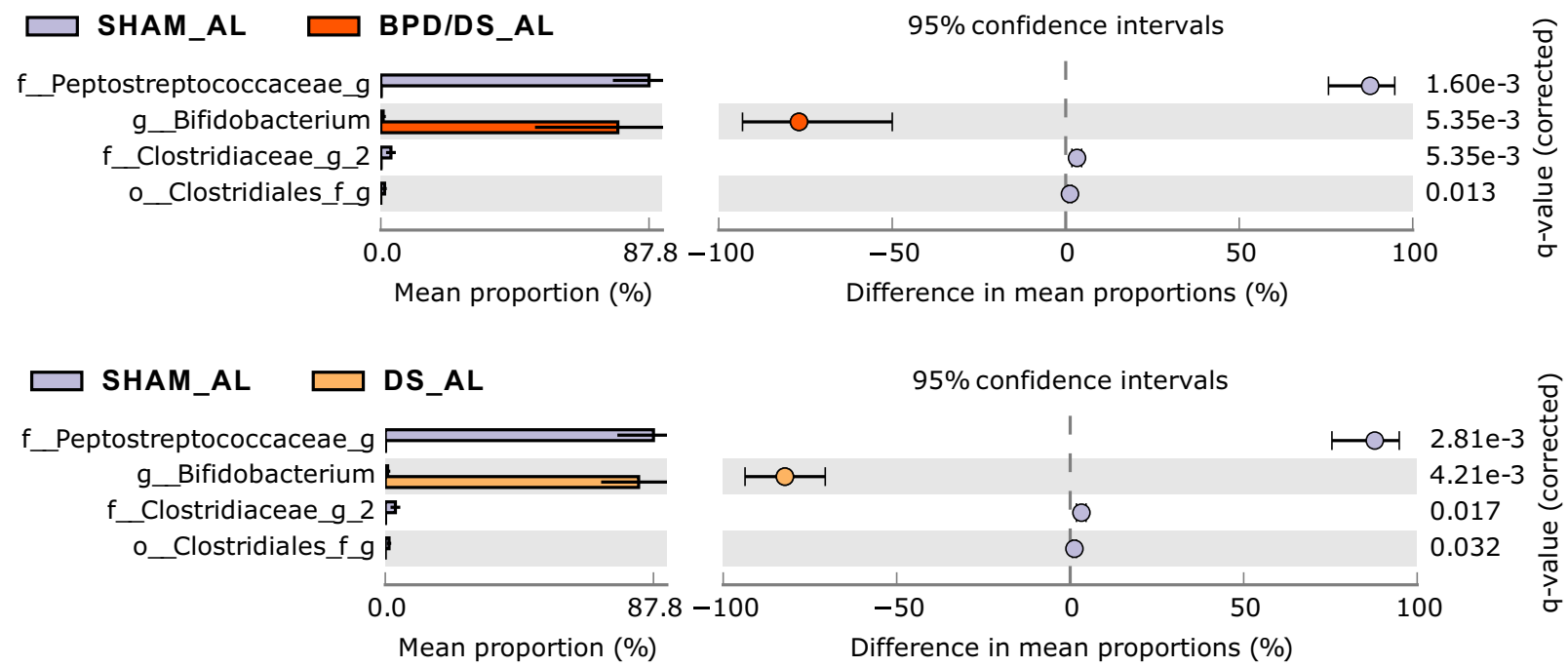

Fig. 4 (continued)

microbiota were only seen in groups with body composition changes, one may thus argue that the microbiota shift following $\mathrm{BPD} / \mathrm{DS}$ is a consequence of the concomitant lower fat gain. Such a possibility appears unlikely as the alteration in the microbiota composition appears specific to BPD/DS; RYGB has been reported to cause weight loss and changes in gut microbiota distinct from that observed in BPD/DS rats [21]. Although a causal relationship between the microbiota shift and beneficial effects of the BPD/DS and DS cannot readily be established, one can reason that the microbiota composition associated with the malabsorptive procedures might confer health benefits. In fact, the increased proportion of bacteria from the Bifidobacteriales order was linked to the increased abundance of reads assigned to the genus Bifidobacterium which may confer health benefits [30-33] to the host. The predominance of Bifidobacterium in the gut microbiota could reduce low-grade inflammation [34] as well as underlying the raised levels of GLP-1 and PYY following BPD/DS [35]. The latter hormones cause satiating effect [36-38], and GLP-1, which is strongly stimulated in BPD/DS animals $[5,6]$, is seen as one of the most potent incretins $[8,9,39]$. One however needs to remain cautious in linking the rise in Bifidobacterium to the beneficial effects of the surgery as one cannot accurately estimate from the present data how much of the increased relative abundance of Bifidobacteriales was due to the drop of Clostridiales versus the true growth in Bifidobacteriales.

Altogether the results of the present study suggest that nutrient malabsorption, while reducing fat deposition and improving glucose metabolism, also alters gut microbiota composition, leading to the beneficial effects of DS and BPD/DS. 
We speculate that the positive outcomes of both surgeries may be enhanced through the modulation of gut microbiota and, more specifically, by the increased proportional abundance of Bifidobacterium throughout the gastrointestinal tract.

\section{Compliance with Ethical Standards}

Conflict of Interest The authors declare that they have no conflict of interest.

Ethical Approval All applicable institutional and/or national guidelines for the care and use of animals were followed. The protocol was approved by the Université Laval Animal Care and Use Committee, and animals were cared according to the Canadian Guide for the Care and Use of Laboratory Animals.

Open Access This article is distributed under the terms of the Creative Commons Attribution 4.0 International License (http:// creativecommons.org/licenses/by/4.0/), which permits unrestricted use, distribution, and reproduction in any medium, provided you give appropriate credit to the original author(s) and the source, provide a link to the Creative Commons license, and indicate if changes were made.

\section{References}

1. World Health Organization. Obesity: Preventing and Managing the Global Epidemic: Report on a WHO consultation. (WHO technical report series 894).Geneva, Switzerland: World Health Organization; 2000.

2. Angrisani L, Santonicola A, Iovino P, et al. Bariatric surgery worldwide 2013. Obes Surg. 2015;25(10):1822-32.

3. Biertho L, Biron S, Hould FS, et al. Is biliopancreatic diversion with duodenal switch indicated for patients with body mass index $<50$ $\mathrm{kg} / \mathrm{m}^{2}$ ? Surg Obes Relat Dis. 2010;6(5):508-14.

4. Biertho L, Lebel S, Marceau S, et al. Laparoscopic sleeve gastrectomy: with or without duodenal switch? A consecutive series of 800 cases. Dig Surg. 2014;31(1):48-54.

5. Li W, Baraboi ED, Cluny NL, et al. Malabsorption plays a major role in the effects of the biliopancreatic diversion with duodenal switch on energy metabolism in rats. Surg Obes Relat Dis. 2015;11(2):356-66.

6. Baraboi ED, Li W, Labbe SM, et al. Metabolic changes induced by the biliopancreatic diversion in diet-induced obesity in male rats: the contributions of sleeve gastrectomy and duodenal switch. Endocrinology. 2015;156(4):1316-29.

7. Sinclair P, Brennan DJ, le Roux CW. Gut adaptation after metabolic surgery and its influences on the brain, liver and cancer. Nat Rev Gastroenterol Hepatol. 2018;15(10):606-24.

8. Jorgensen NB, Jacobsen SH, Dirksen C, et al. Acute and long-term effects of Roux-en-Y gastric bypass on glucose metabolism in subjects with type 2 diabetes and normal glucose tolerance. Am J Physiol Endocrinol Metab. 2012;303(1):122-31.

9. Laferrère B. (2016). Bariatric surgery and obesity: influence on the incretins. Int J Obes Suppl, 6(Suppl 1), S32-S36.

10. Guo Y, Huang ZP, Liu CQ, et al. Modulation of the gut microbiome: a systematic review of the effect of bariatric surgery. Eur $\mathrm{J}$ Endocrinol. 2018;178(1):43-56.

11. Anhê FF, Varin TV, Schertzer JD, et al. The gut microbiota as a mediator of metabolic benefits after bariatric surgery. Can J Diabetes. 2017;41(4):439-47.
12. Magouliotis DE, Tasiopoulou VS, Sioka E, et al. Impact of bariatric surgery on metabolic and gut microbiota profile: a systematic review and meta-analysis. Obes Surg. 2017;27(5):1345-57.

13. Zhang H, DiBaise JK, Zuccolo A, et al. Human gut microbiota in obesity and after gastric bypass. Proc Natl Acad Sci U S A. 2009;106(7):2365-70.

14. Tremaroli V, Karlsson F, Werling M, et al. Roux-en-Y gastric bypass and vertical banded gastroplasty induce long-term changes on the human gut microbiome contributing to fat mass regulation. Cell Metab. 2015;22(2):228-38.

15. Liou AP, Paziuk M, Luevano JM, et al. Conserved shifts in the gut microbiota due to gastric bypass reduce host weight and adiposity. Sci Transl Med. 2013;5(178):178ra141.

16. Nadreau E, Baraboi ED, Samson P, et al. Effects of the biliopancreatic diversion on energy balance in the rat. Int $\mathrm{J}$ Obes. 2006;30(3):419-29.

17. Marceau P, Biron S, Hould FS, et al. Duodenal switch: long-term results. Obes Surg. 2007;17(11):1421-30.

18. Furet JP, Kong LC, Tap J, et al. Differential adaptation of human gut microbiota to bariatric surgery-induced weight loss: links with metabolic and low-grade inflammation markers. Diabetes. 2010;59(12):3049-57.

19. Patrone V, Vajana E, Minuti A, et al. Postoperative changes in fecal bacterial communities and fermentation products in obese patients undergoing bilio-intestinal bypass. Front Microbiol. 2016;7:200.

20. Li JV, Ashrafian H, Bueter M, et al. Metabolic surgery profoundly influences gut microbial-host metabolic cross-talk. Gut. 2011;60(9):1214-23.

21. Shao Y, Ding R, Xu B, et al. Alterations of gut microbiota after Roux-en-Y gastric bypass and sleeve gastrectomy in SpragueDawley rats. Obes Surg. 2017;27(2):295-302.

22. Graessler J, Qin Y, Zhong H, et al. Metagenomic sequencing of the human gut microbiome before and after bariatric surgery in obese patients with type 2 diabetes: correlation with inflammatory and metabolic parameters. Pharm J. 2013;13(6):514-22.

23. Manning S, Pucci A, Batterham RL. Roux-en-Y gastric bypass: effects on feeding behavior and underlying mechanisms. J Clin Invest. 2015;125(3):939-48.

24. Sabate JM, Coupaye M, Ledoux S, et al. Consequences of small intestinal bacterial overgrowth in obese patients before and after bariatric surgery. Obes Surg. 2017;27(3):599-605.

25. Sung YK, Gwak GY, Choi MS, et al. A case of nonalcoholic steatohepatitis and small intestinal bacterial overgrowth with peripheral edema caused by intestinal bypass surgery and relieved by repair. Gut Liver. 2012;6(4):520-3.

26. Hassn A, Luhmann A, Rahmani S, et al. Medium-term results of combined laparoscopic sleeve gastrectomy and modified jejunoileal bypass in bariatric surgery. Obes Surg. 2016;26(10):2316-23.

27. Borbély YM, Osterwalder A, Kroll D, et al. Diarrhea after bariatric procedures: diagnosis and therapy. World J Gastroenterol. 2017;23(26):4689-700.

28. Stefater MA, Perez-Tilve D, Chambers AP, et al. Sleeve gastrectomy induces loss of weight and fat mass in obese rats, but does not affect leptin sensitivity. Gastroenterology. 2010;138(7):2426-36. 2436 e2421-2423

29. Valenti V, Martin M, Ramirez B, et al. Sleeve gastrectomy induces weight loss in diet-induced obese rats even if high-fat feeding is continued. Obes Surg. 2011;21(9):1438-43.

30. Corrêa NB, Péret Filho LA, Penna FJ, et al. A randomized formula controlled trial of Bifidobacterium lactis and Streptococcus thermophilus for prevention of antibiotic-associated diarrhea in infants. J Clin Gastroenterol. 2005;39(5):385-9.

31. Cani PD, Neyrinck AM, Fava F, et al. Selective increases of bifidobacteria in gut microflora improve high-fat-diet-induced diabetes in mice through a mechanism associated with endotoxaemia. Diabetologia. 2007;50(11):2374-83. 
32. Aoki R, Kamikado K, Suda W, et al. A proliferative probiotic Bifidobacterium strain in the gut ameliorates progression of metabolic disorders via microbiota modulation and acetate elevation. Sci Rep. 2017;7:43522.

33. Mangin I, Dossou-Yovo F, Leveque $\mathrm{C}$, et al. Oral administration of viable Bifidobacterium pseudolongum strain Patronus modified colonic microbiota and increased mucus layer thickness in rat. FEMS Microbiol Ecol. 2018;94(11)

34. Sanchis-Chordà J, Del Pulgar EMG, Carrasco-Luna J, et al. Bifidobacterium pseudocatenulatum CECT 7765 supplementation improves inflammatory status in insulin-resistant obese children. Eur J Nutr. 2018;https://doi.org/10.1007/s00394-018-1828-5

35. Bomhof MR, Saha DC, Reid DT, et al. Combined effects of oligofructose and Bifidobacterium animalis on gut microbiota and glycemia in obese rats. Obesity (Silver Spring). 2014;22(3):763-71.

36. le Roux CW, Aylwin SJB, Batterham RL, et al. Gut hormone profiles following bariatric surgery favor an anorectic state, facilitate weight loss, and improve metabolic parameters. Ann Surg. 2006;243(1):108-14.

37. Lee WJ, Chong K, Lin YH, et al. Laparoscopic sleeve gastrectomy versus single anastomosis (mini-) gastric bypass for the treatment of type 2 diabetes mellitus: 5-year results of a randomized trial and study of incretin effect. Obes Surg. 2014;24(9):1552-62.

38. Schmidt JB, Gregersen NT, Pedersen SD, et al. Effects of PYY ${ }_{3-36}$ and GLP-1 on energy intake, energy expenditure, and appetite in overweight men. Am J Physiol Endocrinol Metab. 2014;306(11): 1248-56.

39. Guidone C, Manco M, Valera-Mora E, et al. Mechanisms of recovery from type 2 diabetes after malabsorptive bariatric surgery. Diabetes. 2006;55(7):2025-31.

Publisher's Note Springer Nature remains neutral with regard to jurisdictional claims in published maps and institutional affiliations. 